\title{
Equation Calculating Internal Energy Change Derived from the Clausius Inequality
}

\author{
Chengshu Jin \\ School of Food and Pharmaceutical Engineering, Suihua University, Suihua, Heilongjiang 152061, China \\ Email: jinchengshu_1@163.com
}

\begin{abstract}
In the paper, the relations of heat or work between a reversible process and its realistic process are discussed, which could be applied to the chemical reactions and Rossini and Frandsen experiment and so on. It is proved the ratios of work to temperature and internal energy change to temperature are all the state function in the reversible process, otherwise, the Clausius inequality will be violated. In an isothermal process of reversible Stirling cycle, the internal energy change is equal to zero for the gas. Thus, the new equation calculating the internal energy change has been obtained for the gas, namely that $\mathrm{d} U=C_{V} \mathrm{~d} T$, it is convenient and easy calculative.
\end{abstract}

Keywords: First law, Clausius inequality, chemical reactions, Stirling cycle, thermodynamic function.

\section{Introduction}

The relation of heat, work, and internal energy change will obey the first law of thermodynamics (namely the energy conservation law). In a process, the heat, work, and internal energy change are able to be transferred each other. The Clausius inequality originated from the Carnot theorem and second law of thermodynamics, it is absolute correct [1-8]. Any spontaneous and realistic cycle is similar to the heat engine or refrigeration engine. The entropy generation expresses the difference on the ratio of heat to temperature between a reversible process and its realistic process. The entropy generation concept could be applied to large range [9-11]. The difference on the ratios of work to temperature or internal energy change to temperature between a reversible process and its realistic process have been researched too [12]. In this paper, the relations of heat, work, internal energy change, enthalpy change, and free energy change will be investigated in the chemical reactions and so on. The relation between internal energy change and temperature for the gas in an isothermal process will be studied using the reversible Stirling cycle in text.

\section{Relations among the Heat, Work, and Internal Energy Change}

In a spontaneous or realistic process, we would assume

$$
\begin{aligned}
Q_{\text {real }}(B) & =Q_{r}(A)+Q_{p f}(B) \\
W_{\text {real }}(B) & =W_{r}(A)+W_{p f}(B)
\end{aligned}
$$

where, $Q_{r}(A)$ and $W_{r}(A)$ are the heat and work in a reversible path $(A)$ respectively. $Q_{\text {real }}(B)$ and $W_{\text {real }}(B)$ are the heat and work in a realistic path $(B)$, respectively. $Q_{p f}(B)$ and $W_{p f}(B)$ are the heat and work caused by the irreversible path and friction resistance in any irreversible path $(B)$, respectively. The realistic paths may be the reversible or irreversible paths. The friction resistance does not exist in any reversible process. That is $Q_{r}$ and $W_{r}$ do not contain $Q_{p f}(B)$ and $W_{p f}(B)$. For the gas, $W_{r}(A)$ can be calculated from $p-V$ diagram is easy in the reversible process (often employing the gas equation of state or the state experiment datums), but calculating and measuring the values of $W_{\text {real }}(B)$ isn't easy in the irreversible process (according to the temperature or pressure change path, comparing $W_{\text {real }}(B)$ with $W_{r}(A)$ is not difficult in $p$ - $V$ diagram). A reversible path and its irreversible path are distinction for the gas in a process, otherwise, $W_{p f}(B)$ will be equal to zero. The heat and work are the path functions in some processes. The heat and work are taken as positive if the energy is supplied to the system and negative if the energy is lost out of the system.

According to the first law of thermodynamics, it is obvious that $\Delta U_{r}(A)=Q_{r}(A)+W_{r}(A)$, $\Delta U_{\text {real }}(B)=Q_{\text {real }}(B)+W_{\text {real }}(B)$, and $\Delta U_{r}(A)=\Delta U_{\text {real }}(B)$ in a process, where, $U_{r}(A)$ and $U_{\text {real }}(B)$ are the 
internal energy in a reversible path $(A)$ and its realistic path $(B)$, respectively. The internal energy change $\Delta U$ is the state function. In a process, we can find

$$
Q_{p f}(B)=-W_{p f}(B)
$$

\section{Relations on the Ratios of Heat to Temperature, Work to Temperature and Internal Energy Change to Temperature}

In a spontaneous or realistic process, the Clausius inequality can be rewritten as follow

$$
\int_{i}^{f} \frac{\delta Q_{r}(A)}{T}-\int_{i}^{f} \frac{\delta Q_{r e a l}(B)}{T_{r e s}} \geq 0
$$

where, the equality is for a reversible process, the inequality is for an irreversible process. $\int_{i}^{f} \frac{\delta Q_{r}(A)}{T}$ and $\int_{i}^{f} \frac{\delta Q_{\text {real }}(B)}{T_{\text {res }}}$ are the ratio of heat to temperature in a reversible path $(A)$ and a realistic path $(B)$, respectively. $\Delta S$ and $\int_{i}^{f} \frac{\delta Q_{r}(A)}{T}$ are all the entropy change, that is, $\Delta S=\int_{i}^{f} \frac{\delta Q_{r}(A)}{T}$. In a process, $T_{i}$ and $T_{f}$ are the absolute temperature of system in initial state and final state, respectively. $T$ expresses the absolute temperature of system transforms from $T_{i}$ to $T_{f}$. $T_{\text {res }}$ is the absolute temperature of surroundings or reservoirs, and $T_{\text {res }}$ can vary or influence the absolute temperature of system. Of course, $T_{\text {res }}$ is the same as $T$ in any reversible process or isothermal process. Any spontaneous or realistic process has to obey the Clausius inequality.

$$
\Delta S_{g}=\int_{i}^{f} \frac{\delta Q_{r}(A)}{T}-\int_{i}^{f} \frac{\delta Q_{\text {real }}(B)}{T_{\text {res }}}[10,13], \Delta S_{g} \text { is defined as the entropy generation. Thus, in a }
$$

spontaneous or realistic process, we can gain $\Delta S_{g} \geq 0$. Therefore, $\int_{i}^{f} \frac{\delta Q_{\text {real }}(B)}{T_{\text {res }}}$ is entropy flow. The entropy generation will become into zero for the equilibrium and changeless state.

The relation among the ratios of heat to temperature, work to temperature, and internal energy change to temperature can be given by

$$
\int_{i}^{f} \frac{\mathrm{d} U_{r}(A)}{T}-\int_{i}^{f} \frac{\mathrm{d} U_{r e a l}(B)}{T_{\text {res }}}=\int_{i}^{f} \frac{\delta Q_{r}(A)}{T}-\int_{i}^{f} \frac{\delta Q_{\text {real }}(B)}{T_{r e s}}+\int_{i}^{f} \frac{\delta W_{r}(A)}{T}-\int_{i}^{f} \frac{\delta W_{\text {real }}(B)}{T_{r e s}}
$$

In a spontaneous or realistic process, if $\int_{i}^{f} \frac{\delta W_{r}(A)}{T}-\int_{i}^{f} \frac{\delta W_{\text {real }}(B)}{T_{\text {res }}} \leq 0$, according to Eq. (5), we can obtain

$$
\int_{i}^{f} \frac{\delta Q_{r}(A)}{T}-\int_{i}^{f} \frac{\delta Q_{r e a l}(B)}{T_{\text {res }}} \geq \int_{i}^{f} \frac{\mathrm{d} U_{r}(A)}{T}-\int_{i}^{f} \frac{\mathrm{d} U_{r e a l}(B)}{T_{r e s}}
$$

Therefore, $\int_{i}^{f} \frac{\mathrm{d} U_{r}(A)}{T}-\int_{i}^{f} \frac{\mathrm{d} U_{\text {real }}(B)}{T_{\text {res }}} \geq 0$ obeys the Clausius inequality namely Eq. (4), which will guarantee that the value of $\int_{i}^{f} \frac{\delta Q_{r}(A)}{T}-\int_{i}^{f} \frac{\delta Q_{r e a l}(B)}{T_{r e s}}$ is no less than zero. $\int_{i}^{f} \frac{\mathrm{d} U_{r}(A)}{T}-\int_{i}^{f} \frac{\mathrm{d} U_{r e a l}(B)}{T_{r e s}}<0$ will violate the Clausius inequality, since $\int_{i}^{f} \frac{\delta Q_{r}(A)}{T}-\int_{i}^{f} \frac{\delta Q_{r e a l}(B)}{T_{r e s}}$ will be more than negative value. If $\int_{i}^{f} \frac{\delta W_{r}(A)}{T}-\int_{i}^{f} \frac{\delta W_{r e a l}(B)}{T_{r e s}}>0$, we have

$$
\int_{i}^{f} \frac{\mathrm{d} U_{r}(A)}{T}-\int_{i}^{f} \frac{\mathrm{d} U_{r e a l}(B)}{T_{r e s}}>\int_{i}^{f} \frac{\delta Q_{r}(A)}{T}-\int_{i}^{f} \frac{\delta Q_{r e a l}(B)}{T_{r e s}}
$$


On the basis of Eq. (4), we can gain $\int_{i}^{f} \frac{\mathrm{d} U_{r}(A)}{T}-\int_{i}^{f} \frac{\mathrm{d} U_{r e a l}(B)}{T_{r e s}}>0 \cdot \int_{i}^{f} \frac{\mathrm{d} U_{r}(A)}{T}-\int_{i}^{f} \frac{\mathrm{d} U_{r e a l}(B)}{T_{r e s}}<0$ will infringe the Clausius inequality. So that, $\int_{i}^{f} \frac{\mathrm{d} U_{r}(A)}{T}-\int_{i}^{f} \frac{\mathrm{d} U_{r e a l}(B)}{T_{r e s}}>0$ is right, it will guarantee that the value of $\int_{i}^{f} \frac{\delta Q_{r}(A)}{T}-\int_{i}^{f} \frac{\delta Q_{\text {real }}(B)}{T_{\text {res }}}$ is no less than zero.

In a spontaneous or realistic process, according to Eq. (5) and $\int_{i}^{f} \frac{\mathrm{d} U_{r}(A)}{T}-\int_{i}^{f} \frac{\mathrm{d} U_{r e a l}(B)}{T_{r e s}} \geq 0$, we have

$$
\int_{i}^{f} \frac{\delta Q_{r}(A)}{T}-\int_{i}^{f} \frac{\delta Q_{r e a l}(B)}{T_{\text {res }}} \geq-\left(\int_{i}^{f} \frac{\delta W_{r}(A)}{T}-\int_{i}^{f} \frac{\delta W_{\text {real }}(B)}{T_{r e s}}\right)
$$

Therefore, $\int_{i}^{f} \frac{\delta W_{r}(A)}{T}-\int_{i}^{f} \frac{\delta W_{\text {real }}(B)}{T_{\text {res }}} \leq 0$ obeys the Clausius inequality namely Eq. (4), which will guarantee that the value of $\int_{i}^{f} \frac{\delta Q_{r}(A)}{T}-\int_{i}^{f} \frac{\delta Q_{\text {real }}(B)}{T_{\text {res }}}$ is no less than zero. $\int_{i}^{f} \frac{\delta W_{r}(A)}{T}-\int_{i}^{f} \frac{\delta W_{\text {real }}(B)}{T_{\text {res }}}>0$ will violate the Clausius inequality, since $\int_{i}^{f} \frac{\delta Q_{r}(A)}{T}-\int_{i}^{f} \frac{\delta Q_{r e a l}(B)}{T_{r e s}}$ will be more than negative value. So that, $\int_{i}^{f} \frac{\delta W_{r}(A)}{T}-\int_{i}^{f} \frac{\delta W_{\text {real }}(B)}{T_{r e s}} \leq 0$ is correct, it will guarantee that the value of $\int_{i}^{f} \frac{\delta Q_{r}(A)}{T}-\int_{i}^{f} \frac{\delta Q_{r e a l}(B)}{T_{r e s}}$ is no less than zero.

Therefore, the difference on the ratios of work to temperature or internal energy change to temperature between a reversible process and its realistic process can be given by

$$
\begin{gathered}
\int_{i}^{f} \frac{\delta W_{r}(A)}{T}-\int_{i}^{f} \frac{\delta W_{\text {real }}(B)}{T_{\text {res }}} \leq 0 \\
\int_{i}^{f} \frac{\mathrm{d} U_{r}(A)}{T}-\int_{i}^{f} \frac{\mathrm{d} U_{\text {real }}(B)}{T_{\text {res }}} \geq 0
\end{gathered}
$$

where, $\int_{i}^{f} \frac{\delta W_{r}(A)}{T}$ or $\int_{i}^{f} \frac{\delta W_{\text {real }}(B)}{T_{\text {res }}}$ are the ratio of work to temperature in a reversible path $(A)$ or a realistic path $(B)$, respectively. $\int_{i}^{f} \frac{\delta W_{r}(A)}{T}$ is defined with $\Delta S_{W}$, namely that $\Delta S_{W}=\int_{i}^{f} \frac{\delta W_{r}(A)}{T}$. $\int_{i}^{f} \frac{\mathrm{d} U_{r}(A)}{T}$ or $\int_{i}^{f} \frac{\mathrm{d} U_{r e a l}(B)}{T_{r e s}}$ are the ratio of internal energy change to temperature in a reversible path $(A)$ or its realistic path $(B)$, respectively. $\int_{i}^{f} \frac{\mathrm{d} U_{r}(A)}{T}$ is defined with $\Delta S_{U}$, namely that $\Delta S_{U}=\int_{i}^{f} \frac{\mathrm{d} U_{r}(A)}{T}$.

If $\left(\Delta S_{W}\right)_{g}=\int_{i}^{f} \frac{\delta W_{r}(A)}{T}-\int_{i}^{f} \frac{\delta W_{r e a l}(B)}{T_{r e s}}$ in a spontaneous or realistic process, we can have $\left(\Delta S_{W}\right)_{g} \leq 0$. The value of $\left(\Delta S_{W}\right)_{g}$ will also change into zero at the equilibrium and changeless state for the spontaneous or realistic processes. Eq. (6) is always criterion for the spontaneous or realistic processes except that any work doesn't exist.

If $\left(\Delta S_{U}\right)_{g}=\int_{i}^{f} \frac{\mathrm{d} U_{r}(A)}{T}-\int_{i}^{f} \frac{\mathrm{d} U_{r e a l}(B)}{T_{r e s}}$ in a spontaneous or realistic process, we shall obtain $\left(\Delta S_{U}\right)_{g} \geq 0$. The value of $\left(\Delta S_{U}\right)_{g}$ will change into zero at the equilibrium and changeless state for the spontaneous or realistic processes. Eq. (7) is always criterion for spontaneous or realistic processes except that the isothermal process.

If a reversible process has two paths namely that the path $(A)$ and path $(B)$, we have 


$$
\begin{gathered}
\int_{i}^{f} \frac{\delta Q_{r}(A)}{T}=\int_{i}^{f} \frac{\delta Q_{r}(B)}{T} \\
\int_{i}^{f} \frac{\mathrm{d} U_{r}(A)}{T}-\int_{i}^{f} \frac{\mathrm{d} U_{r}(B)}{T}=\int_{i}^{f} \frac{\delta W_{r}(A)}{T}-\int_{i}^{f} \frac{\delta W_{r}(B)}{T}
\end{gathered}
$$

Accordingly, $\Delta S_{W}$ and $\Delta S_{U}$ are the state function in the reversible path $(A)$ and path $(B)$, namely that $\int_{i}^{f} \frac{\delta W_{r}(A)}{T}=\int_{i}^{f} \frac{\delta W_{r}(B)}{T}$ and $\int_{i}^{f} \frac{\mathrm{d} U_{r}(A)}{T}=\int_{i}^{f} \frac{\mathrm{d} U_{r}(B)}{T}$. Otherwise, the equations (6, 7) will be infringed. Certainly, the above-mentioned conclusion is reliable and reasonable. For example, $\Delta S_{W}$ and $\Delta S_{U}$ are all the state function for the ideal gas in a reversible process. The van der Waals equation of state does not obey the aforementioned conclusion, therefore, it is an approximate equation. Attentively, " $\mathrm{d}$ " and " $\delta$ " are total differential symbols to the state function and path function respectively. " $\Delta$ " expresses a change of quantity value, "V" is integral symbol.

\section{Results and Discussion}

\subsection{Change Law in the Free Expansion of Ideal Gas}

If the ideal gas in the container of constant volume is freely expanding to another vacuum container of constant volume, see figure 1. Gay-lussac or Joule experiment all told us the temperature would not have any change in aforesaid process. In the realistic isothermal path $(B)$, the temperature does not have any change, thus, the internal energy change is equal to zero for the ideal gas, namely that $\Delta U=0$. Because $W_{\text {real }}(B)=0$, we have $Q_{\text {real }}(B)=0$. The reversible process for path $(A)$ should belong to an isothermal expansion process, the result is

$$
\Delta S=\int_{i}^{f} \frac{\delta Q_{r}(A)}{T}=-\int_{i}^{f} \frac{\delta W_{r}(A)}{T}=n R \ln \frac{V_{f}}{V_{i}} \geq 0
$$

where, $V$ is volume, $R$ is the gas constant, $n$ is the amount of substance, $V_{f}>V_{i}$, as shown in figure $1 . \Delta S_{g}$ is equal to $\Delta S,\left(\Delta S_{W}\right)_{g}$ is equal to the negative value of $\Delta S$, and $\left(\Delta S_{U}\right)_{g}$ is always equal to zero.

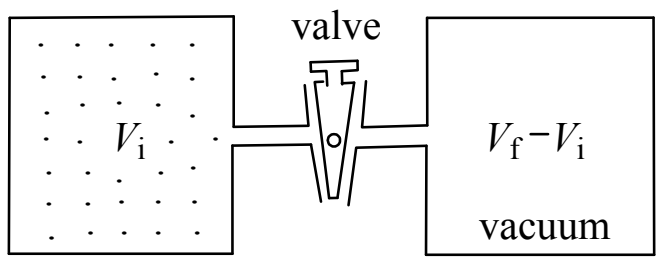

total volume: $V_{\mathrm{f}}$

Figure 1. Free expansion of ideal gas.

\subsection{Change Law in the Chemical Reactions}

For the chemical reactions in a reversible isothermal and constant pressure path $(A)$, we can obtain $Q_{r}(A)=T \Delta S$. If the non-expansion work (namely the other work) exists in a reversible isothermal and constant pressure path $(C)$, we shall have $W_{r}(C)=\Delta U-Q_{r}(C)=\Delta U-T \Delta S$ and $\Delta S_{g}=T \Delta S(A)-Q_{r}(C)=0$, where, $Q_{r}(C)=T \Delta S$. $W_{\max }$ expresses other work in a reversible process, $p \Delta V$ is the pressure-volume work (namely the expansion work), therefore, $W_{r}(C)=W_{\max }(C)-p \Delta V$, the following equations can be given by

$$
\begin{gathered}
W_{\max }(C)=W_{r}(C)+p \Delta V=\Delta U+p \Delta V-T \Delta S=\Delta G \\
\Delta U=W_{\max }(C)-p \Delta V+T \Delta S
\end{gathered}
$$

where, $\Delta H=\Delta U+p \Delta V, \Delta G=\Delta H-T \Delta S, \Delta H$ is the enthalpy change, $\Delta G$ is free energy change. 
If the non-expansion work still exists for the chemical reactions in a realistic isothermal and constant pressure path $(B)$ (temperature may be non-constant, but $T_{i}=T_{f}=T_{\text {res }}$ ), on the basis of Eq. (2), we can obtain

$$
W_{\text {real }}(B)=W_{r}(A)+W_{p f}(B)=\Delta U-Q_{r}(A)+W_{p f}(B)=\Delta U-T \Delta S+W_{p f}(B)
$$

where, $W_{\text {other }}$ expresses the other work in a realistic process, therefore, $W_{\text {real }}(B)=W_{\text {other }}(B)-p \Delta V$. According to Eq. (3), we have

$$
W_{p f}(B)=W_{\text {real }}(B)-\Delta U+T \Delta S=W_{\text {other }}(B)-p \Delta V-\Delta U+T \Delta S=W_{\text {other }}(B)-\Delta G=-Q_{p f}(B)
$$

Then, on the basis of the equations $(8,10)$, we can obtain

$$
W_{\text {other }}(B)=\Delta G+W_{p f}(B)=W_{\max }(C)+W_{p f}(B)
$$

According to the equations $(1,10), Q_{r}(A)=T \Delta S, \Delta G=\Delta H-T \Delta S, Q_{r}(C)=T \Delta S$, we can gain

$$
Q_{\text {real }}(B)=Q_{r}(A)+Q_{p f}(B)=\Delta H-W_{\text {other }}(B) \text { or } Q_{\text {real }}(B)=Q_{r}(C)+Q_{p f}(B)=\Delta H-W_{\text {other }}(B)
$$

Thus, we have

$$
T \Delta S_{g}=T \Delta S-Q_{\text {real }}(B)=T \Delta S+W_{\text {other }}(B)-\Delta H=W_{\text {other }}(B)-\Delta G \geq 0
$$

Thereby, on the basis of the equations $(10,12,13), \Delta H=\Delta U+p \Delta V$, we can gain

$$
\begin{gathered}
W_{\text {other }}(B) \geq \Delta G \text { or } W_{p f}(B)=-Q_{p f}(B) \geq 0 \\
\Delta U=W_{\text {other }}(B)-p \Delta V+Q_{\text {real }}(B)
\end{gathered}
$$

Attentively, if the non-expansion work does not exist in a realistic constant pressure path $(B)$, according to Eq. (10), we can obtain $W_{p f}(B)=-\Delta G=-Q_{p f}(B)$.

\subsection{Internal Energy Change in the Reversible Stirling Cycle}

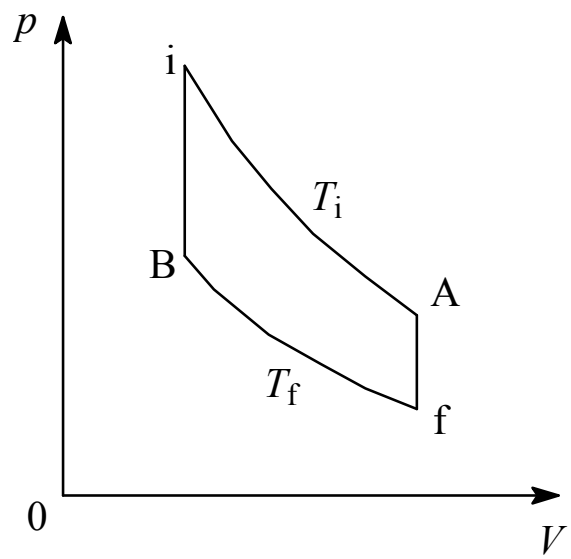

Figure 2. The reversible Stirling cycle.

In the reversible Stirling cycle (see figure 2), the path " $i \rightarrow A$ " and path " $f \rightarrow B$ " are all the reversible isothermal process, and their temperatures are $T_{i}$ and $T_{f}$ respectively. The path " $B \rightarrow i$ " and path " $A \rightarrow f$ " are all the reversible constant volume process. $W_{i B}$ and $W_{f A}$ express the works in the path " $B \rightarrow i$ " and path " $A \rightarrow f$ " are all equal to zero. $\Delta U_{A i}, \Delta U_{f A}, \Delta U_{B f}$, and $\Delta U_{i B}$ express the internal energy change in the path " $i \rightarrow A$ ", path " $A \rightarrow f$ ", path " $f \rightarrow B$ ", and path " $B \rightarrow i$ ", respectively. The equation calculating the internal energy change for the gas can be given by $[1]$

$$
\mathrm{d} U=C_{V} \mathrm{~d} T+\left[T\left(\frac{\partial p}{\partial T}\right)_{V}-p\right] \mathrm{d} V
$$

where, the subscript $V$ in $\left(\frac{\partial p}{\partial T}\right)_{V}$ item indicates constant volume, " $\partial$ " is partial differential symbols, $C_{V}$ is the heat capacity at constant volume. For the gas, we can obtain $\Delta U_{f A}=-\Delta U_{i B}$. So that, $\Delta U_{A i}=-\Delta U_{B f}$. Thus, 
we have $\oint \frac{\mathrm{d} U_{r}}{T}=\int_{i}^{A} \frac{\mathrm{d} U_{A i}}{T_{i}}+\int_{A}^{f} \frac{\mathrm{d} U_{f A}}{T}+\int_{f}^{B} \frac{\mathrm{d} U_{B f}}{T_{f}}+\int_{B}^{i} \frac{\mathrm{d} U_{i B}}{T}=\left(\frac{1}{T_{i}}-\frac{1}{T_{f}}\right) \Delta U_{A i}=0$ in the reversible process $\left(\Delta S_{U}\right.$ is the state function). We know $\left(\frac{1}{T_{i}}-\frac{1}{T_{f}}\right) \neq 0$, therefore, $\Delta U_{A i}$ and $\Delta U_{B f}$ should be all equal to zero. So that, a conclusion is the internal energy change has to be equal to zero for the gas in the isothermal process.

Because the expansion gas in the Rossini and Frandsen experiment [14] belongs to an isothermal and constant pressure process, therefore, $\Delta U=0$. The Rossini and Frandsen experiment is made by two systems namely that the gas system and water system. In the reversible process, Eq. (9) is obeyed by the Rossini and Frandsen experiment, therefore, $T \Delta S=p \Delta V-W_{\max }(C)$ and $\Delta H=p \Delta V$. In the realistic process, Eq. (15) is obeyed by the Rossini and Frandsen experiment, thus, $Q_{\text {real }}(B)=p \Delta V-W_{\text {other }}(B)$ and $\Delta H=p \Delta V$. Attention, the electrical work belongs to $W_{\text {other }}$ in the Rossini and Frandsen experiment. In the isothermal process, measuring the value of heat is easy for the chemical reactions, but the system of expansion gas is open in the Rossini and Frandsen experiment, so the heat cannot be accurately determined. Therefore, it is regretful that the Rossini and Frandsen experiment could not prove the internal energy change having a relation with the pressure and volume for the gas. The figure 3 is a type of the Rossini and Frandsen experiment equipment.

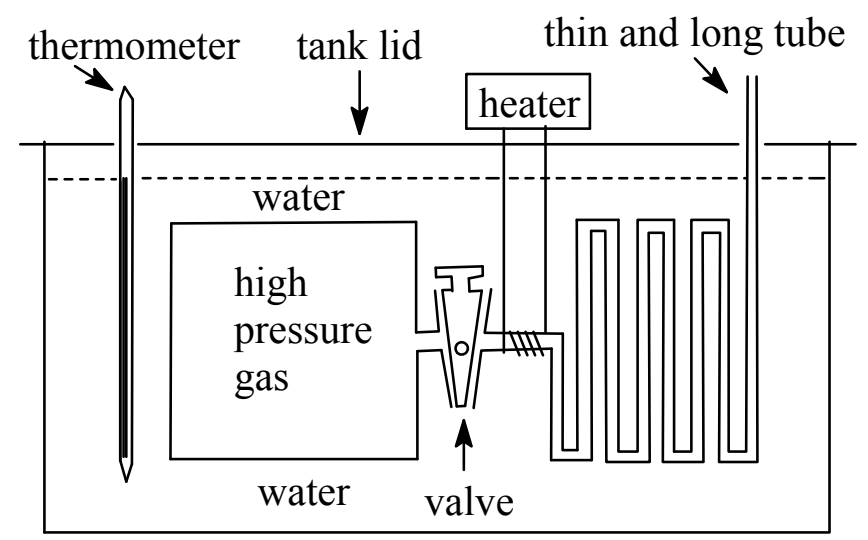

Figure 3. A type of the Rossini and Frandsen experiment equipment. The high pressure real gas slowly expands to reach the air through the valve, the water temperature will keep constant heated by heater in trough.

\section{Conclusion}

For the gas, the internal energy change in any isothermal processes is equal to zero, namely that, $\left(\frac{\partial U}{\partial V}\right)_{T}=0$ and $\left(\frac{\partial U}{\partial p}\right)_{T}=0$ (where, the subscript $T$ indicates constant temperature). Correspondingly, Eq. (16) is not right and disobeys the Clausius inequality. For the gas, Eq. (16) will become into

$$
\mathrm{d} U=C_{V} \mathrm{~d} T
$$

The solid and liquid obey Eq. (17) too.

If the other work, latent heat, and chemical reactions do not exist, we can obtain $\delta Q=C_{V} \mathrm{~d} T+p \mathrm{~d} V$ and $T \mathrm{~d} S=C_{V} \mathrm{~d} T+p \mathrm{~d} V($ where, $\mathrm{d} U=T \mathrm{~d} S-p \mathrm{~d} V)$.

In the constant pressure, the internal energy change for the phase transition or chemical reactions is given by $\Delta U_{c}=\Delta H_{c}-p \Delta V$, where, $\Delta U_{c}$ is the internal energy change in the phase transition or chemical reactions, $\Delta H_{c}$ is the latent heat or chemical reaction heat.

On the basis of Eq. (17), Eq. (7) can be proven to be correct. 
In an irreversible process, if any work doesn't exist, $\left(\Delta S_{W}\right)_{g}$ is equal to zero. In the isothermal process, $\left(\Delta S_{U}\right)_{g}$ is equal to zero. In a reversible process, $\Delta S_{g},\left(\Delta S_{W}\right)_{g}$, and $\left(\Delta S_{U}\right)_{g}$ are all equal to zero, but it is impossible that $\Delta U, Q_{r}, W_{r}, \Delta S, \Delta S_{W}$, and $\Delta S_{U}$ are all equal to zero.

In the equilibrium and changeless state, $\Delta U, Q, W, \Delta S, \Delta S_{W}, \Delta S_{U}, \Delta S_{g},\left(\Delta S_{W}\right)_{g}$, and $\left(\Delta S_{U}\right)_{g}$ are all equal to zero.

In the many irreversible non-isothermal, non-constant volume, and non-constant pressure processes, calculating and measuring the values of $Q_{\text {real }}(B), W_{\text {real }}(B), \int_{i}^{f} \frac{\delta Q_{\text {real }}(B)}{T_{\text {res }}}$, and $\int_{i}^{f} \frac{\delta W_{\text {real }}(B)}{T_{\text {res }}}$ is very difficult for the gas. Calculating the values of Eq. (7) may not use any gas equation of state. In the isothermal process, Eq. (7) isn't criterion, but the equations $(4,6)$ are all criteria.

\section{References}

1. C. Borgnakke, R. E. Sonntag, Fundamentals of thermodynamics, 8th ed., John Wiley \& Sons Singapore Pte Ltd, Singapore, 2014, pp. 35-496.

2. P. Atkins, J. de Paula, Atkins' Physical chemistry, 10th ed., Oxford University Press, Oxford, 2014, pp. 45-130.

3. S. Lee, K. Lee, J. Lee, "An alternative presentation of the second law of thermodynamics", Journal of Chemical Education, vol. 9, no. 4, pp. 771-773, 2015. http://dx.doi.org/10.1021/ed5007822.

4. H. B. Callen, Thermodynamics and an introduction to thermostatics, Second ed., John Wiley, New York, 1985, pp. 103-109.

5. W. Muschik, "Contact temperature and internal variables: A glance back, 20 years later", Journal of Non-Equilibrium Thermodynamics, vol. 39, no. 3, pp. 113-121, 2014. http://dx.doi.org/10.1515/jnet-2014-0016.

6. S. I. Sandler, L. V. Woodcock, "Historical observations on laws of thermodynamics", Journal of Chemical 8 Engineering Data, vol. 55, no. 10, pp. 4485-4490, 2010. http://dx.doi.org/10.1021/je1006828.

7. E. A. Gislason, N. C. Craig, "Criteria for spontaneous processes derived from the global point of view", Journal of Chemical Education, vol. 90, no. 5, pp. 584-590, 2013. http://dx.doi.org/10.1021/ed300570u.

8. H. DeVoe, "A comparison of local and global formulations of thermodynamics", Journal of Chemical Education, vol. 90, no. 5, pp. 591-597, 2013. http://dx.doi.org/10.1021/ed300497j.

9. Z. Zhang, C. Drapaca, Z. Zhang, S. Zhang, S. Sun, H. Liu, "Leakage evaluation by virtual entropy generation (VEG) Method", Entropy, vol. 20, no. 1, pp. 14, 2018. http://dx.doi.org/10.3390/e20010014.

10. I. Dincer, Y. A. Cengel, "Energy, entropy and exergy concepts and their roles in thermal engineering", Entropy, vol. 3, pp. 116-149, 2001. http://dx.doi.org/10.3390/e3030116.

11. U. Lucia, "Quanta and entropy generation", Physica A: Statistical Mechanics and its Applications, vol. 419, pp. 115-121, 2015. http://dx.doi.org/10.1016/j.physa.2014.10.040

12. C. Jin, "Studies on equalities and inequalities of heat, work and ratio of work to temperature", International Journal of Physical Sciences, vol. 12, no. 17, pp. 205-210, 2017. http://dx.doi.org/10.5897/IJPS2017.4624.

13. G. Grazzini, R. Borchiellini, and U. Lucia, "Entropy versus entransy", Journal of Non-Equilibrium Thermodynamics, vol. 38, pp. 259-271, 2013. http://dx.doi.org/10.1515/jnetdy-3013-0008.

14. F. D. Rossini, and M. Frandsen, "The calorimetric determination of the intrinsic energy of gases as a function of the pressure. Data on oxygen and its mixtures with carbon dioxide to 40 atmospheres at $28^{\circ} \mathrm{C}$ ", Bureau of Standards Journal of Research, vol. 9, pp. 733-747, 1932. 